Research Article

\title{
Comparative Transcriptomic Analysis of Root Cadmium Responses in Two Chinese Rice Cultivars Yuzhenxiang and Xiangwanxian 12
}

\author{
Shangdu Zhang $\mathbb{D}^{1,2}$ Zhenliang Luo, ${ }^{3}$ Xiang $W u,{ }^{2}$ Bangzhi Shi, ${ }^{2}$ Huidan Jiang, \\ Leliang Zhou, ${ }^{2}$ and Lianyang Bai $i^{1,4}$ \\ ${ }^{1}$ Long Ping Branch of Graduate School, Central South University, Changsha, Hunan 410125, China \\ ${ }^{2}$ Guizhou Rice Research Institute, Guizhou Academy of Agricultural Sciences, Guiyang, Guizhou 550006, China \\ ${ }^{3}$ Hunan Rice Research Institute, Hunan Academy of Agricultural Sciences, Changsha, Hunan 410125, China \\ ${ }^{4}$ Hunan Agricultural Biotechnology Research Institute, Hunan Academy of Agricultural Sciences, Changsha, \\ Hunan 410125, China
}

Correspondence should be addressed to Lianyang Bai; lybai@hunaas.cn

Received 4 November 2021; Revised 25 November 2021; Accepted 27 November 2021; Published 13 December 2021

Academic Editor: Wenneng Wu

Copyright (c) 2021 Shangdu Zhang et al. This is an open access article distributed under the Creative Commons Attribution License, which permits unrestricted use, distribution, and reproduction in any medium, provided the original work is properly cited.

\begin{abstract}
Cadmium $(\mathrm{Cd})$ pollution in paddy soil is an increasingly serious issue in rice production. It has been reported that there is a higher or lower grain Cd accumulation in the rice cultivars Yuzhenxiang (YZX) or Xiangwanxian 12 (XWX), respectively. To better manage the Cd pollution problem, the genes that might play vital roles in governing the difference in root Cd responses between these two rice cultivars were examined. In this study, the results of RNA sequencing (RNA-seq) showed that there were 341 and 161 differentially expressed genes in the roots of YZX and XWX after Cd exposure, respectively. Among these genes, 7 genes, such as Os06g0196300 (OsJ_019618), Os07g0570700 (OsJ_24808), ADI1, GDCSH, HSFB2C, PEX11-4, and CLPB1, possessed higher degree nodes with each other, through interaction analysis by the STRING (search tool for the retrieval of interacting genes/ proteins) software, suggesting that they might play vital roles in Cd response. Based on GO enrichment analysis, 41 differently expressed genes after Cd treatment in YZX or XWX were identified to be related to Cd response. Through comparative transcriptomic analysis, 257 genes might be associated with the root Cd response difference between YZX and XWX. Furthermore, we supposed that ADI1, CFBP1, PEX11-4, OsJ_019618, OsJ_24808, GDCSH, CLPB1, LAC6, and WNK3 might be implicated in Cd response based on the combined analysis of RT-qPCR, interaction, and GO annotation analysis. In conclusion, the numerous genes that might be related to Cd stress response and root Cd response difference between YZX and XWX at the booting stage may be of benefit for the development of rice varieties with low $\mathrm{Cd}$ consumption.
\end{abstract}

\section{Introduction}

Rice (Oryza sativa L.) is the primary source of calorie intake in plenty of countries, including China, and the staple food of over half of the world's population $[1,2]$. To meet the food demand of increasing world populations, it is imperative to improve the rice yield [3]. However, the sustainability and productivity of the rice production system are constantly threatened by multiple factors, such as environmental pollution, climate change, and excessive use of pesticides and fertilizers [2].
Heavy metal pollution is the predominant type in all soil contaminants, and cadmium $(\mathrm{Cd})$ contamination ranks the first among heavy metal contaminants in China $[4,5]$. Cd contamination, mainly caused by mining, e-waste, and overuse of nitrogen and phosphate fertilizers, has been found in large-scale agricultural soil in China [6, 7]. Cd is readily absorbed by rice plants and easily transferred to food chains [8]. Moreover, it is often accumulated in rice grains and human bodies due to its long half-life of up to 25-30 years $[8,9]$. Excessive Cd exposure not only can inhibit the 
growth of rice plants but also can decrease the quality/ nutrients/yields of rice grains [7]. Additionally, the intake of Cd-contaminated rice can markedly increase the risks of multiple diseases, such as cancer, osteoporosis, and liver/ kidney injury, as well as nervous system diseases [9-11]. To reduce the potential harm of $\mathrm{Cd}$ on human health, it is imperative to screen out the genes that play vital roles in $\mathrm{Cd}$ response in rice plants [7].

It has been reported that there is a notable difference in $\mathrm{Cd}$ content in the grains of different rice cultivars, which might be caused by the genotypic and environmental diversities among rice cultivars $[12,13]$. More specifically, rice $\mathrm{Cd}$ concentration is closely associated with heading time, soil $\mathrm{pH}$, mutation/dysregulation/diversity of multiple genes, or quantitative trait locus related to $\mathrm{Cd}$ response (absorption, translocation, and accumulation) among different rice subspecies and cultivars $[12,13]$. Over the past decades, high-throughput RNA sequencing (RNA-seq) in combination with function annotation/ enrichment and bioinformatics prediction analysis has been widely used to decipher or speculate the essential genes/biological processes/pathways under different conditions at the transcriptomic level and gain a deep and comprehensive understanding of molecular basis underlying the phenotypic/biological differences in plants including rice [14-16].

In this study, RNA-seq-based transcriptomic analysis of root samples of YZX and XWX with or without Cd treatment at the booting stage was performed to identify key genes in root Cd response in Yuzhenxiang (YZX, a high Cd accumulation rice cultivar) and Xiangwanxian 12 (XWX, a low $\mathrm{Cd}$ accumulation rice cultivar) and genes associated with root Cd accumulation difference between YZX and XWX. RNA-seq results showed that 341 and 161 transcripts were differentially expressed in the roots of YZX or XWX rice after Cd treatment. Moreover, the protein-protein interaction (PPI) networks of dysregulated transcripts in response to Cd exposure in YZX or XWX were established by the STRING database and 7 genes that might play vital roles in the response to Cd pollution were screened out based on the node degrees of proteins in the PPI networks. Additionally, 41 transcripts that function as crucial players in Cd response were filtered out based on GO annotation analysis. Furthermore, 257 transcripts that might be associated with the difference in root Cd response between YZX or XWX were screened out by comparative transcriptomic analysis. Also, we further examined the expression patterns of 10 genes (ferredoxin-1 (ADI1), fructose-1, 6-bisphosphatase (CFBP1), glycine cleavage system $\mathrm{H}$ protein (GDCSH), laccase-6 (LAC6), peroxiredoxin Q (Os06g0196300), ribosome-recycling factor (Os07g0570700), peroxisomal membrane protein 11-4 (PEX11-4), probable serine/threonineprotein kinase $\mathrm{WNK} 3$, chaperone protein CLPB1, and heat stress transcription factor B-2c (HSFB2C)) by RT-qPCR assay and filtered some key genes related to $\mathrm{Cd}$ stimulation based on RNA-seq/RT-qPCR/interaction/GO enrichment/ GO annotation analysis together with previous study outcomes.

\section{Materials and Methods}

2.1. Plant Materials and Treatment. Two Indica rice cultivars (i.e., YZX and XWX) were cultivated in the LT-36VL climatic growth chamber (Percival, Perry, IA, USA) at the Hunan Agricultural Biotechnology Research Institute (Changsha, China). In the preliminary tests, YZX and XWX rice at 4 different growth phases (i.e., seedling, tillering, booting, and grain-filling stages) were cultivated in a hydroponic system containing $2 \mathrm{mg} / \mathrm{L}$ of $\mathrm{Cd}$ under low-temperature $\left(15-20^{\circ} \mathrm{C}\right)$, moderate-temperature $\left(22-27^{\circ} \mathrm{C}\right)$, and high-temperature $\left(30-35^{\circ} \mathrm{C}\right)$ condition for $48 \mathrm{~h}$. Cd concentration was determined according to the instructions in the Chinese National Standard GB 5009.15-2014.

2.2. RNA Sequencing. In the RNA-seq and real-time quantitative reverse transcription PCR (RT-qPCR) validation experiments, YZX and XWX rice were grown at $22-27^{\circ} \mathrm{C}$ (moderate temperature) in the hydroponic system with or without $2 \mathrm{mg} / \mathrm{L}$ of Cd exposure for $48 \mathrm{~h}$. Next, rice root samples were collected. The samples were divided into 4 groups: AMD group (YZX group with Cd treatment), AMK group (YZX group without Cd treatment), BMD group (XWX group with Cd addition), and BMK group (XWX group without $\mathrm{Cd}$ exposure). Each group contains 3 root samples.

RNA was extracted from the root samples using the TRIzol reagent (Thermo Scientific, Waltham, MA, USA) according to the protocols of the manufacturer. The concentration, purity, and quality of RNA were examined by NanoDrop 2000 spectrophotometer (Thermo Scientific). RNA sequencing was carried out as previously described [17]. The cDNA library was constructed using the TruSeq RNA sample preparation kit (Illumina, San Diego, CA, USA) following the protocols of the manufacturer. The mRNA with poly-A structure was enriched by Poly-Toligo-attached magnetic beads (Illumina). The cDNA library was sequenced using the PE150 strategy by Biomarker Technologies Co., Ltd. (Beijing, China). Raw sequencing data were processed and filtered into high-quality clean data. Next, the clean data were aligned to the reference genome of Oryza sativa L. subsp. Indica using HISAT2 and then assembled and quantified using StringTie. The genome version used in the sequence assembly was ASM465v1. (http://plants.ensembl. org/Oryza_indica/Info/Index).

2.3. Differential Expression Analysis. Differential expression analysis was performed using the DESeq R package. Genes were defined to be differentially expressed at the fold change $\geq 2$ and FDR $<0.01$.

2.4. Gene Enrichment Analysis. KEGG enrichment analysis was performed using the KOBAS3 database (http://kobas. cbi.pku.edu.cn/kobas3/genelist/). $P<0.05$ represented that the terms were significantly enriched. 
2.5. Interaction Network Construction. The interaction networks of gene-coding proteins were constructed by the STRING database (https://www.string-db.org/).

2.6. $R T-q P C R$ Assay. RNA was reversely transcribed into first-strand cDNA without genomic DNA contamination using TransScript One-Step gDNA Removal and cDNA Synthesis SuperMix (TransGen Biotech Co., Ltd, Beijing, China). Briefly, RNA was coincubated with Oligo (dT) 18 primer for $5 \mathrm{~min}$ at $65^{\circ} \mathrm{C}$ and then immediately placed on an ice bath for $2 \mathrm{~min}$. Then, the reaction mixture was coincubated with TS Reaction Mix, TransScript RT/RI Enzyme Mix, and gDNA Remover at $42^{\circ} \mathrm{C}$ for $30 \mathrm{~min}$ and then terminated at $85^{\circ} \mathrm{C}$ for $5 \mathrm{~min}$. Next, cDNA was amplified and quantified using the ChamQ Universal SYBR qPCR Master Mix (Vazyme Biotech Co., Ltd., Nanjing, China) and specific quantitative primers under the following thermocycling conditions: $95^{\circ} \mathrm{C}$ for $3 \mathrm{~min}, 40$ cycles of $95^{\circ} \mathrm{C}$ for $10 \mathrm{~s}$, and $60^{\circ} \mathrm{C}$ for $30 \mathrm{~s}$. 18sRNA served as the housekeeping gene to normalize the expression of other genes. RT-qPCR was performed on Roche LightCycler 480 II Real-Time PCR Detection System (Basel, Sweden). The quantitative primer sequences are presented in Table 1. The RNA samples in the RT-qPCR assay were identical to those used in RNA-seq experiments.

2.7. Statistical Analysis. Data analysis was performed using GraphPad Prism 7 software (GraphPad Software, Inc., San Diego, CA, USA). Results were displayed as mean \pm standard deviation. The difference among groups was examined using one-way ANOVA and Dunnett post hoc test. Statistically significant differences were set at $P<0.05$.

2.8. Accession Numbers. The raw data of RNA-seq reads were deposited in the National Center for Biotechnology Information (NCBI) database under the accession number (Submission ID: SUB10609471, BioProject ID: PRJNA777353). Biosample accessions were as follows: SAMN22851327, SAMN22851328, SAMN22851329, SAMN22851330, SAMN22851331, SAMN22851332, SAMN22851333, SAMN22851334, SAMN22851335, SAMN22851336, SAMN22851337, and SAMN22851338.

\section{Results}

3.1. Cd Accumulation Difference in the Roots of YZX and XWX at Four Different Growth Stages. Previous studies have reported that there is a higher Cd accumulation in the first node under panicle, panicle node, and grains of YZX cultivar compared to XWX cultivar $[16,18]$. We supposed that the difference in grain $\mathrm{Cd}$ accumulation in these two rice cultivars might be caused by the discrepancy of root Cd absorptive ability. Moreover, root $\mathrm{Cd}$ uptake capacity has been found to be varied at different growth temperatures and growth stages in rice [19-21]. To identify the optimal conditions under which the difference of $\mathrm{Cd}$ concentration in the roots of YZX and XWX was the most prominent, the concentrations of $\mathrm{Cd}$ in the roots of these two rice cultivars at the seedling/tillering/booting/grain-filling stage under low/moderate/high temperature and $2 \mathrm{mg} / \mathrm{L}$ of $\mathrm{Cd}$ stress were measured. Results showed that there was a noticeable difference in root $\mathrm{Cd}$ content at the booting stage when these two rice cultivars were cultured under the moderate-temperature condition (Figure 1). Hence, YZX and XWX rice were cultivated at moderate temperature and $2 \mathrm{mg} / \mathrm{L}$ of $\mathrm{Cd}$ stress for $48 \mathrm{~h}$ to explore the gene expression alterations in response to $\mathrm{Cd}$ treatment in the following experiments.

\subsection{Identification of Differentially Expressed Transcripts after} $C d$ Treatment in YZX and XWX. In this study, RNA-seq technology was used to identify Cd response-related transcripts in YZX and XWX and investigate genes that might play vital roles in governing the difference in root $\mathrm{Cd}$ accumulation between YZX and XWX. The statistics information of clean data after filtration of RNA-seq raw data is shown in Table 2. The GC content of clean data was approximately $50 \%$ (Table 2). Moreover, more than $94 \%$ of bases have a recognition accuracy of over $99.9 \%$ in the clean data (Table 2). Differential expression analysis showed that 341 transcripts were differentially expressed (206 downregulated and 135 upregulated) in the AMD vs. AMK group (Supplementary Figure 1(a) and Supplementary Table 1). Also, 75 downregulated transcripts and 86 upregulated transcripts were identified in the BMD vs. BMK group (Supplementary Figure 1(b) and Supplementary Table 2). Moreover, 18 dysregulated transcripts (i.e., BGIOSGA022091, BGIOSGA007908, BGIOSGA007302, BGIOSGA029201, BGIOSGA022060, BGIOSGA023662, BGIOSGA026675, BGIOSGA001422, BGIOSGA025476, BGIOSGA030712, BGIOSGA004724, BGIOSGA018872, BGIOSGA010590, BGIOSGA001025, BGIOSGA016478, BGIOSGA020263, BGIOSGA001117, and BGIOSGA 000917) in the AMD vs. AMK group and 6 dysregulated transcripts (i.e., BGIOSGA004809, BGIOSGA030712, BGIOSGA034657, BGIOSGA012380, BGIOSGA018872, and BGIOSGA020584) in the BMD vs. BMK group were identified to be involved in inorganic ion transport and metabolism based on COG_class and KOG_class annotation analyses in Supplementary Tables 1 and 2. These transcripts might play vital roles in governing the difference in root $\mathrm{Cd}$ accumulation in YZX and XWX.

3.3. Construction of PPI Networks of Dysregulated Genes in the $A M D$ vs. AMK and BMD vs. BMK Groups. Next, the STRING database was used to investigate the relationships of proteins encoded by the abovementioned differentially expressed genes. The interaction networks of the dysregulated genes in the AMD vs. AMK group are presented in Supplementary Figure 2(a) and Supplementary Table 3. Similarly, the interaction relationships of the differentially expressed genes in the BMD vs. BMK group are shown in Supplementary Figure 2(b) and Supplementary Table 4. Additionally, the node degree (number of interacted proteins) of each protein in the PPI network of Supplementary Figures 2(a) and 2(b) was analyzed and is displayed in Supplementary Tables 5 and 
TABLE 1: Quantitative primer sequences of 10 interested genes.

\begin{tabular}{|c|c|c|}
\hline Gene ID & Primer name & Primer $\left(5^{\prime}-3^{\prime}\right)$ \\
\hline 18s rRNA & $\begin{array}{l}\text { 18s rRNA-F1 } \\
\text { 18s rRNA-R1 }\end{array}$ & $\begin{array}{l}\text { CTACGTCCCTGCCCTTTCTACA } \\
\text { ACACTTCACCGGACCATTCAA }\end{array}$ \\
\hline BGIOSGA029403 & $\begin{array}{l}\text { HSFB2C-F1 } \\
\text { HSFB2C-R1 }\end{array}$ & $\begin{array}{l}\text { ACAACTTCTCCAGCTTCGTG } \\
\text { ACCTTCCGGCGGTGTATATC }\end{array}$ \\
\hline BGIOSGA016829 & $\begin{array}{l}\text { PEX11-4-F1 } \\
\text { PEX11-4-R1 }\end{array}$ & $\begin{array}{l}\text { TCGTGAGCTGTTCCAACTCT } \\
\text { TGCCAGTGAGCTGTTCAGTA }\end{array}$ \\
\hline BGIOSGA017714 & $\begin{array}{l}\text { CLPB1-F1 } \\
\text { CLPB1-R1 }\end{array}$ & $\begin{array}{l}\text { GTTCGAAGAACGGCTCAAGG } \\
\text { ACGAGGTGTATCTCGTCGAT }\end{array}$ \\
\hline BGIOSGA015634 & $\begin{array}{l}\text { WNK3-F1 } \\
\text { WNK3-R1 }\end{array}$ & $\begin{array}{l}\text { CGTCGACTTCATGTCATCGG } \\
\text { TGCAAATGCTGCTCCTCTTC }\end{array}$ \\
\hline BGIOSGA033278 & $\begin{array}{l}\text { GDCSH-F1 } \\
\text { GDCSH-R1 }\end{array}$ & $\begin{array}{c}\text { AGGACGGGTGGATGATCAAG } \\
\text { AGAGCCTAGTGAGCGTCTTC }\end{array}$ \\
\hline BGIOSGA004865 & $\begin{array}{l}\text { CFBP1-F1 } \\
\text { CFBP1-R1 }\end{array}$ & $\begin{array}{l}\text { GGGAAGTATTGCGTGTGCTT } \\
\text { AGGCTGTAACACGTCCTCAA }\end{array}$ \\
\hline BGIOSGA027798 & $\begin{array}{l}\text { ADI1-F1 } \\
\text { ADI1-R1 } \\
\end{array}$ & $\begin{array}{l}\text { TCATCGAGACCCACAAGGAG } \\
\text { AGCATAGGGACGACGACATT }\end{array}$ \\
\hline BGIOSGA004807 & $\begin{array}{l}\text { LAC6-F1 } \\
\text { LAC6-R1 } \\
\end{array}$ & $\begin{array}{l}\text { TGTCAGCCAGGGAATACGAG } \\
\text { GGACTGTCACGTTGTATGGC }\end{array}$ \\
\hline BGIOSGA025975 & $\begin{array}{l}\text { Os07g0570700-F1 } \\
\text { Os07g0570700-R1 }\end{array}$ & $\begin{array}{l}\text { GTTCCAGAACGCCCAAACAT } \\
\text { GGCATGCCTCAATACTGCTC }\end{array}$ \\
\hline BGIOSGA021734 & $\begin{array}{l}\text { Os06g0196300-F1 } \\
\text { Os06g0196300-R1 }\end{array}$ & $\begin{array}{l}\text { ACAAGGTGAGGAAGGAGTGG } \\
\text { GGAGGATCTTGAGGGTCTCG }\end{array}$ \\
\hline
\end{tabular}

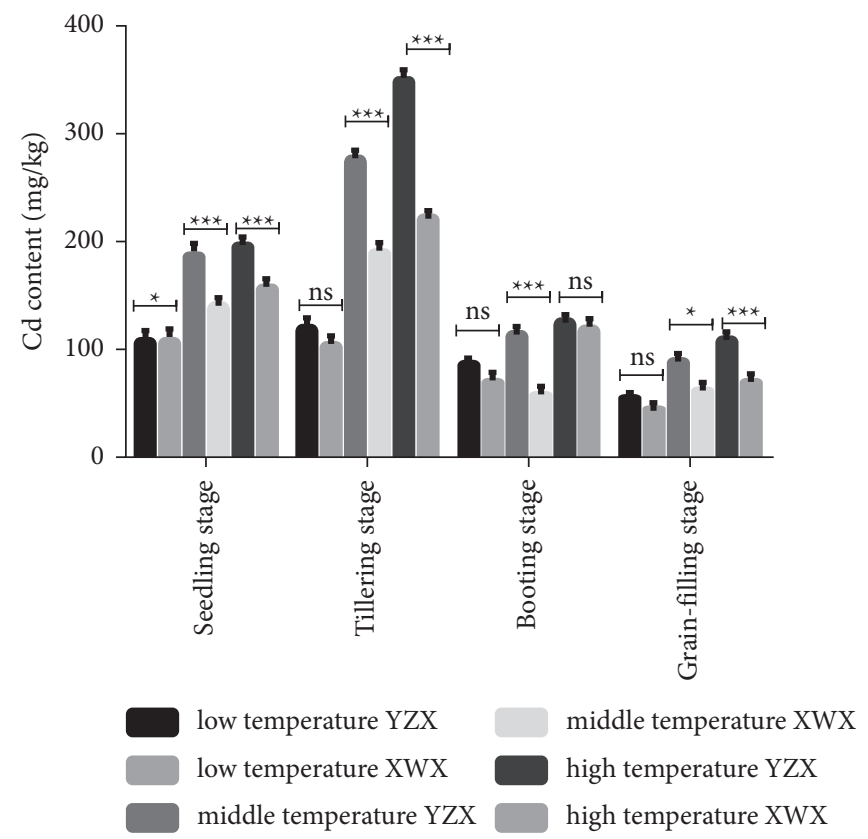

FIgURE 1: Cd accumulation difference in the roots of YZX and XWX at 4 different growth stages under different temperatures and the same Cd stress. YZX and XWX rice at different growth stages (i.e., seedling, tillering, booting, and grain-filling stages) were cultured for $48 \mathrm{~h}$ under different temperatures (low temperature $\left(15-20^{\circ} \mathrm{C}\right)$, moderate temperature $\left(22-27^{\circ} \mathrm{C}\right)$, or high temperature $\left(30-35^{\circ} \mathrm{C}\right)$ ) and $2 \mathrm{mg} / \mathrm{L} \mathrm{of} \mathrm{Cd}$ stress. Next, Cd concentrations in the roots of these two rice cultivars were determined.

6 , respectively. Genes with greater node degrees might play central roles in the response to $\mathrm{Cd}$. Among genes with higher node degrees, 7 genes (Os06g0196300 (OsJ_019618), Os07g0570700 (OsJ_24808), ADI1, GDCSH, HSFB2C, PEX11-4, and CLPB1) were screened out for further
RT-qPCR validation. Moreover, interaction analysis of dysregulated genes in the AMD vs. AMK group revealed that there was a complex interaction among OsJ_019618, OsJ_24808, ADI1, GDCSH, and PEX11-4. For instance, OsJ_019618 could interact with OsJ_24808, ADI1, GDCSH, 
TABLE 2: The statistics information of clean data.

\begin{tabular}{|c|c|c|c|c|}
\hline Sample no. & Clean reads & Clean bases & GC content (\%) & $\% \geq Q 30$ \\
\hline AMD-1 & $28,320,921$ & $8,469,123,354$ & 52.51 & 94.89 \\
\hline AMD-2 & $24,166,384$ & $7,224,223,020$ & 52.62 & 95.04 \\
\hline AMD-3 & $23,919,699$ & $7,147,482,544$ & 52.16 & 95.05 \\
\hline AMK-1 & $25,675,783$ & $7,673,764,994$ & 52.99 & 94.99 \\
\hline AMK-2 & $24,904,107$ & $7,449,500,706$ & 51.75 & 95.23 \\
\hline AMK-3 & $23,973,697$ & $7,172,841,032$ & 52.79 & 94.77 \\
\hline BMD-1 & $30,635,040$ & $9,159,932,340$ & 53.28 & 96.41 \\
\hline BMD-2 & $21,090,058$ & $6,311,270,668$ & 51.64 & 94.84 \\
\hline BMD-3 & $23,708,848$ & $7,088,906,522$ & 51.67 & 94.81 \\
\hline BMK-1 & $25,223,039$ & $7,541,950,044$ & 52.28 & 94.88 \\
\hline BMK-2 & $37,274,481$ & $11,138,840,832$ & 53.13 & 96.36 \\
\hline BMK-3 & $27,498,859$ & $8,216,506,798$ & 52.49 & 96.44 \\
\hline
\end{tabular}

Notes. Clean reads: the total number of paired-end reads in clean data; clean bases: the total number of bases in clean data; GC content: the percentages of G and $\mathrm{C}$ bases in clean data; $\geq \mathrm{Q} 30 \%$ : the percentage of bases with the quality value $\geq 30$ (base identification accuracy $\geq 99.9 \%$ ).

or PEX11-4 (Supplementary Tables 3 and 5). ADI1 could interact with OsJ_019618, GDCSH, or PEX11-4 (Supplementary Tables 3 and 5). Moreover, there was a potential interaction between OsJ_24808 and OsJ_019618 or GDCSH (Supplementary Tables 3 and 5).

3.4. Comparative Analysis of Cd-Responsive Genes in XWX and YZX. GO annotation analysis of the 341 differentially expressed transcripts in the AMD vs. AMK group showed that 37 transcripts were involved in the response to $\mathrm{Cd}$ (Supplementary Table 7). Moreover, 23 of the 161 differentially expressed transcripts in the BMD vs. BMK group were identified to be implicated in $\mathrm{Cd}$ response based on GO annotation analysis of dysregulated transcripts in the BMD vs. BMK group (Supplementary Table 8). Among these Cdresponsive transcripts, 19 common elements were found in AMD vs. AMK and BMD vs. BMK groups (Figure 2(a) and Supplementary Table 9). These common transcripts had the same alteration trends in the AMD vs. AMK and BMD vs. BMK groups (Supplementary Table 9). And 18 or 4 transcripts were found to be differentially expressed only in the AMD vs. AMK group or BMD vs. BMK group, respectively (Figure 2(a) and Supplementary Table 9). These 22 transcripts might have crucial roles in regulating the difference in root Cd accumulation between YZX and XWX at the booting stage. Additionally, we noticed that 43 transcripts were differentially expressed only in the BMD vs. BMK group, but not notably altered in the AMD vs. AMK group (Supplementary Table 10). Also, 214 transcripts were found to be markedly upregulated or downregulated in the AMD vs. AMK group, whereas the difference in the expression of these 214 transcripts was not significant in the BMD vs. BMK group (Supplementary Table 11). The 257 transcripts in Supplementary Tables 10 and 11 are integrated into Supplementary Table 12 . We believed that these transcripts might also be related to the difference in root $\mathrm{Cd}$ accumulation between XWX and YZX. Also, transcripts with notably different upregulated or downregulated ratios in the BMD vs. BMK and AMD vs. AMK groups might be implicated in root $\mathrm{Cd}$ accumulation difference between two rice cultivars, which were not analyzed in our project. KEGG enrichment analysis showed that the transcripts in Supplementary Table 12 mainly participated in the regulation of metabolic pathways, biosynthesis of secondary metabolites, carbon fixation in photosynthetic organisms, carbon metabolism, glutathione metabolism, fructose and mannose metabolism, glycolysis/gluconeogenesis, starch and sucrose metabolism, and glycerophospholipid metabolism (Supplementary Table 13 and Figure 2(b)). For instance, $23,16,5,7$, or 5 differentially expressed genes were significantly enriched in KEGG pathways related to metabolism, biosynthesis of secondary metabolites, carbon fixation in photosynthetic organisms, carbon metabolism, or glutathione metabolism, respectively (Supplementary Table 13). The top 20 KEGG pathways are shown in Figure 2(b).

3.5. Expression Analysis of 10 Interested Genes by RT-qPCR Assay. Next, 10 genes including the 7 abovementioned genes with greater node degrees in the PPI networks, 1 interested gene (LAC6), and 2 genes related to heavy metal stress tolerance (WNK3 and CFBP1) (detailed information is shown in the Discussion section) were selected for further RT-qPCR validation. RT-qPCR results showed that the expression levels of ADI1, CFBP1, GDCSH, LAC6, Os06g0196300, Os07g0570700, PEX11-4, and WNK3 were notably downregulated in both AMD vs. AMK and BMD vs. BMK groups (Figure 3(a), 3(b), 3(d), and 3(f)-3(j)). CLPB1 and $\mathrm{HSFB} 2 \mathrm{C}$ were highly expressed in the AMD vs. AMK group, but not notably changed in the BMD vs. BMK group (Figures 3(c) and 3(e)). Comparative analysis of RT-qPCR and RNA-seq outcomes disclosed that the alteration trends of these 10 genes were consistent in response to Cd treatment in YZX and XWX rice cultivars, although some differences were not statistically significant (Figures $3(a)-3(j)$ ).

3.6. Interaction and Enrichment Analysis of These 10 Genes. The relationships of these 10 genes were further deciphered by PPI analysis via the STRING database. Results suggested that 7 gene-encoded proteins (ADI1, CFBP1, PEX11-4, OsJ_019618, OsJ_24808, GDCSH, and CLPB1) could form a potential regulatory network (Figure 4). The interaction 

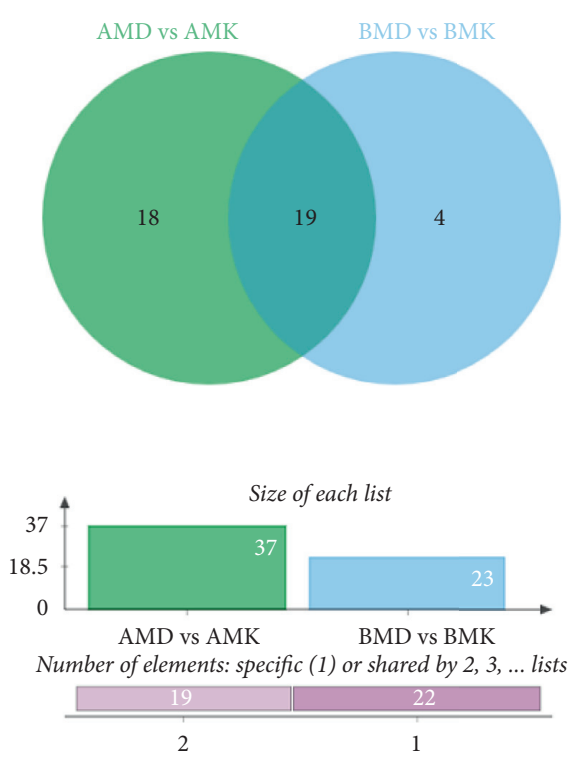

(a)

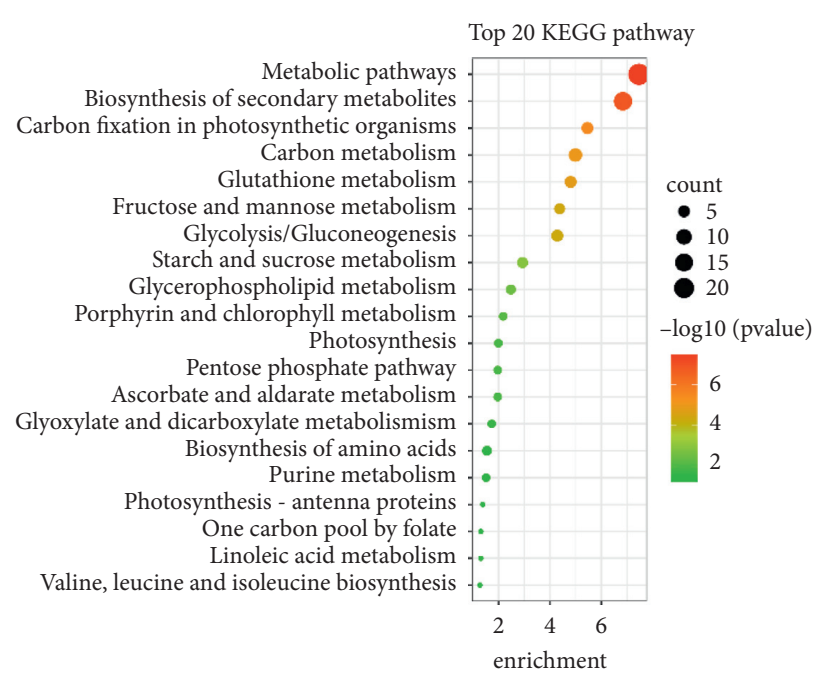

(b)

FIgURe 2: Comparative analysis of Cd-responsive genes in XWX and YZX. (a) Venn analysis for transcripts in Supplementary Tables 7 and 8. AMD vs. AMK list: differentially expressed transcripts related to Cd response in the AMD vs. AMK group based on GO annotation analysis of dysregulated transcripts in the AMD vs. AMK group. BMD vs. BMK list: differentially expressed transcripts related to Cd response in the BMD vs. BMK group based on GO annotation analysis of dysregulated transcripts in the BMD vs. BMK group. Upper subfigure: Venn diagrams showing a number of differentially expressed transcripts related to Cd response in the AMD vs. AMK and BMD vs. BMK groups, as well as the number of overlapping genes. Middle subfigure: number of transcripts in the AMD vs. AMK and BMD vs. BMK lists. Bottom subfigure: number of specific (1) and shared (2) transcripts in the AMD vs. AMK and BMD vs. BMK lists. (b) The top 20 KEGG pathways enriched by the transcripts in Supplementary Table 12. The circle size denotes the number of transcripts in corresponding KEGG pathway terms. The circle color represents the " $-\log _{10}(P$ value $)$ ".

analysis of these 10 proteins is shown in sheet 1 of Supplementary Table 14. Moreover, GO enrichment analysis revealed that 7 genes (LAC6, CFBP1, OsJ_019618, WNK3, OsJ_24808, ADI1, and GDCSH) were enriched in the metabolic process (Supplementary Table 14, sheet 2). Additionally, 6 genes (LAC6, CFBP1, CLPB1, WNK3, OsJ_24808, and ADI1) had a potential ion binding activity (Supplementary Table 14, sheet 3). Furthermore, annotation analysis revealed that 3 genes (LAC6, OsJ_019618, and PEX11-4) were involved in the regulation of the response to oxidative stress (Supplementary Table 14, sheet 4).

3.7. Correlation Analysis of These 10 Genes and Cd Response. As shown in Supplementary Table 5, OsJ_019618, OsI_24808, ADI1, GDCSH, PEX11-4, CLPB1, and HSFB2C could interact with 59,44,36,29, 17, 6, and 6 genes in the interaction network of dysregulated genes in the AMD vs. AMK group, respectively. And CLPB1, PEX11-4, and HSFB2C could interact with 6,5 , and 3 genes in the BMD vs. BMK group, respectively (Supplementary Table 6). To explore the association of these genes and Cd response, Venn analysis of genes that could interact with the 10 abovementioned genes and GO-annotated Cd-related genes in Supplementary Tables 7 and 8 was performed. Results showed that OsJ_019618 could interact with 4 genes related to the Cd response (i.e., APX2, HCF136, GLN2, and ALDP). ADI1 could interact with 2 Cd-related genes (i.e., ALDP and GLN2). OsJ_24808, GDCSH, or CLPB1 could interact with
Cd-responsive gene TPI, GLN2, or HSP17.4, respectively. These data further suggested the close association of these genes and $\mathrm{Cd}$ response.

\section{Discussion}

Rice is a big reservoir of potentially toxic heavy metals in paddy soil-rice systems $[22,23]$. The contamination of heavy metals in soil not only influences rice growth and grain yields but also seriously threatens the health of animals and humans that consume rice [22-24]. In this study, genes related to Cd response and root $\mathrm{Cd}$ content difference between YZX and XWX were further examined in the roots of these two rice cultivars with different grain $\mathrm{Cd}$ accumulation.

Both RT-qPCR and RNA-seq outcomes suggested that 10 genes (i.e., PEX11-4, CLPB1, HSFB2C, LAC6, CFBP1, OsJ_019618, WNK3, OsJ_24808, ADI1, and GDCSH) might play vital roles in the response to Cd in both YZX and XWX. Moreover, interaction analysis suggested that 7 genes (ADI1, CFBP1, PEX11-4, OsJ_019618, OsJ_24808, GDCSH, and CLPB1) could form a potential regulatory network in rice. Additionally, OsJ_019618 could interact with 4 GO-annotated genes related to $\mathrm{Cd}$ response (i.e., APX2, HCF136, ALDP, and GLN2). Moreover, there was a potential interaction between $\mathrm{ADI} 1$ and $\mathrm{GO}$-annotated Cd-related gene ALDP or GLN2. OsJ_24808, CLPB1, or GDCSH could interact with GO-annotated Cd-responsive gene TPI, HSP17.4, or GLN2, respectively. Furthermore, APX2 

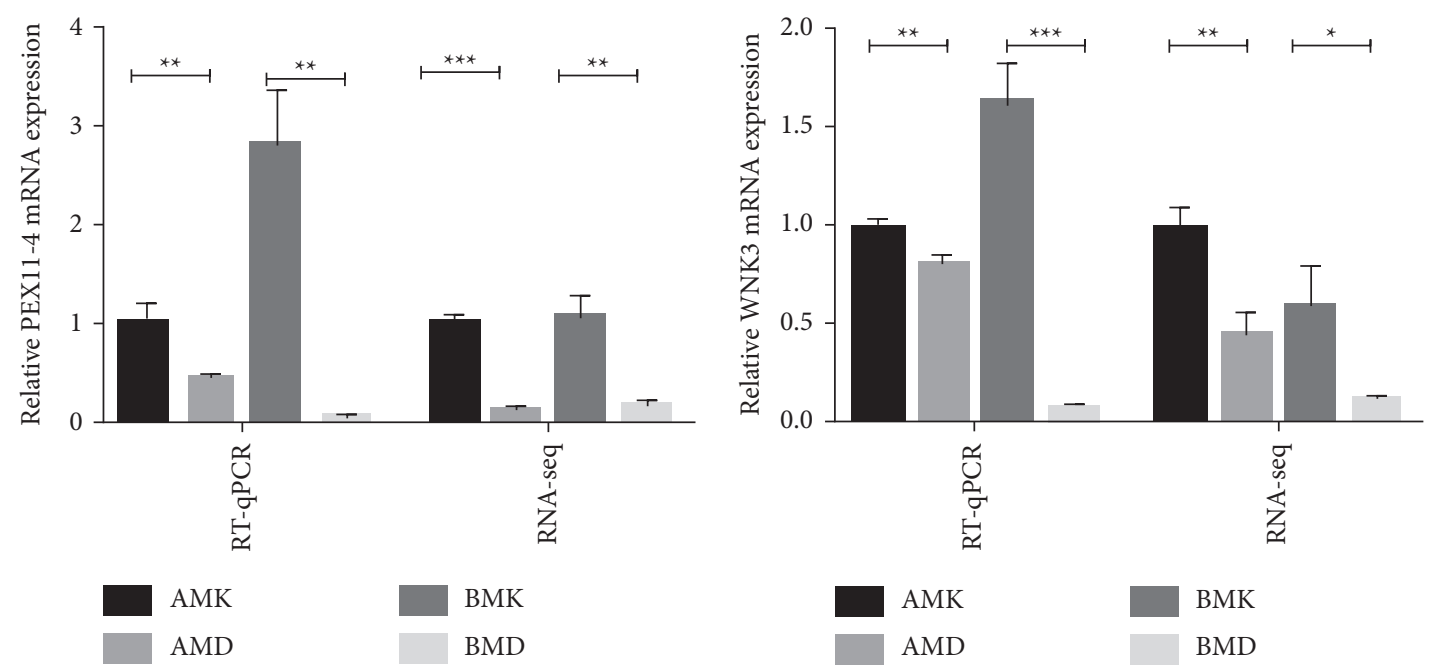

(a)

(b)
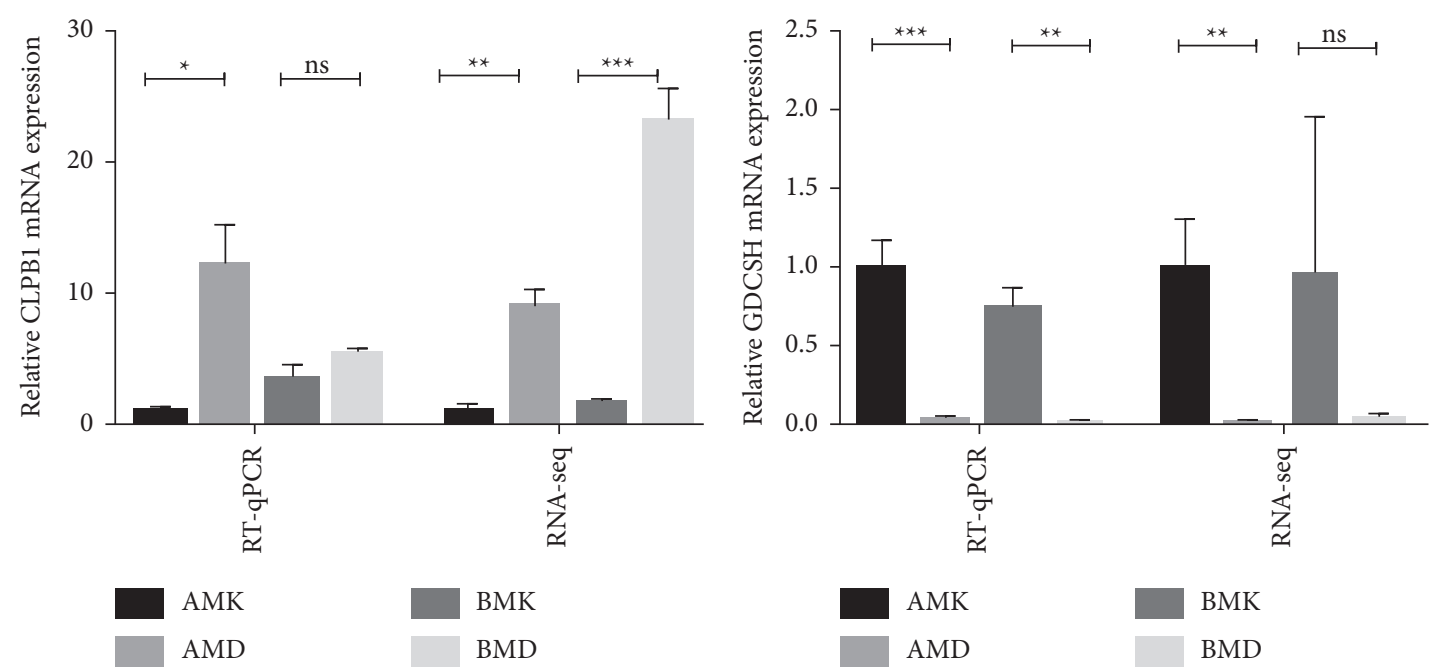

(c)
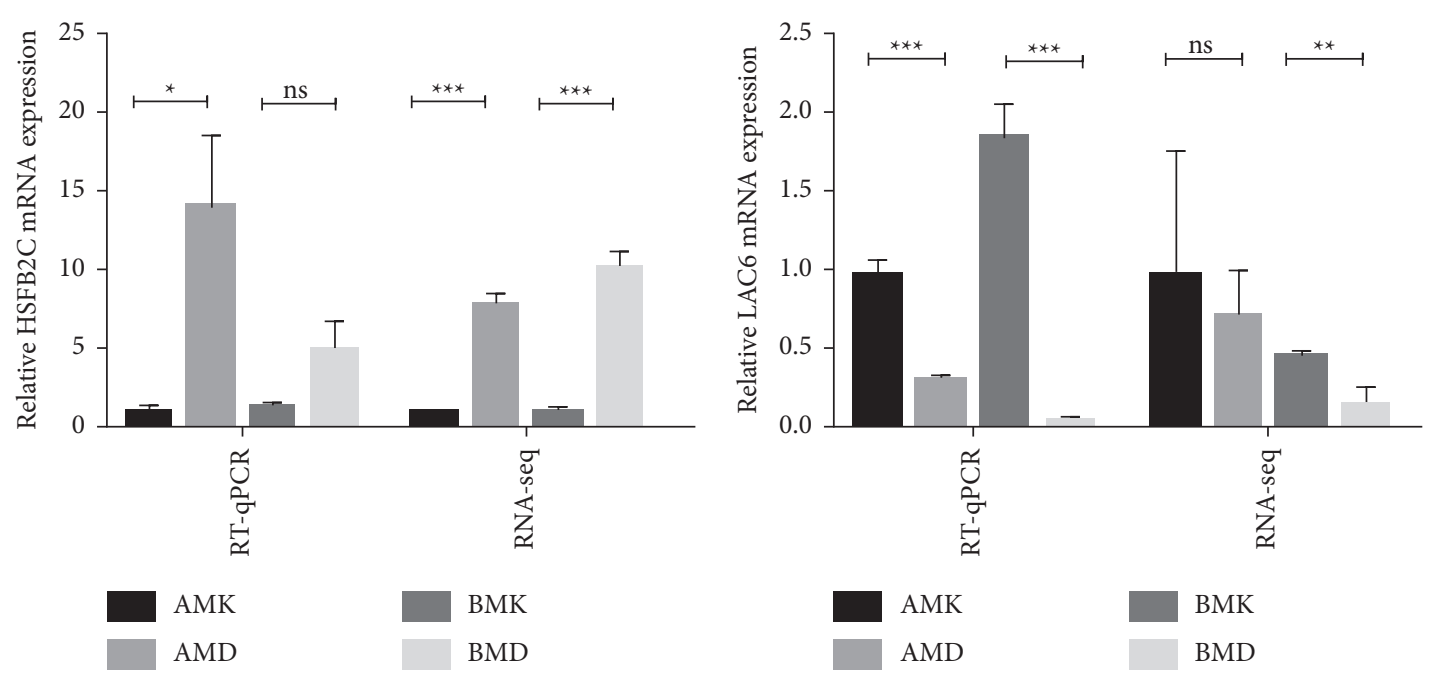

(e)

(f)

Figure 3: Continued. 


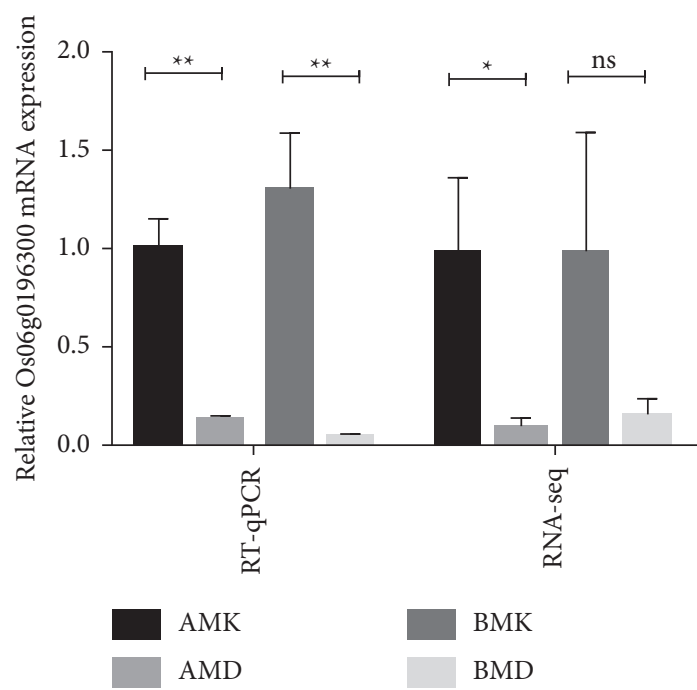

(g)

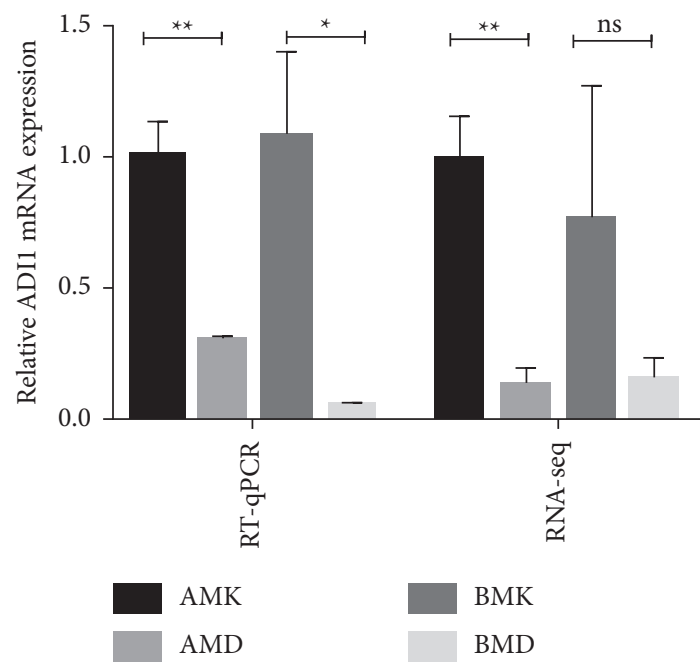

(i)

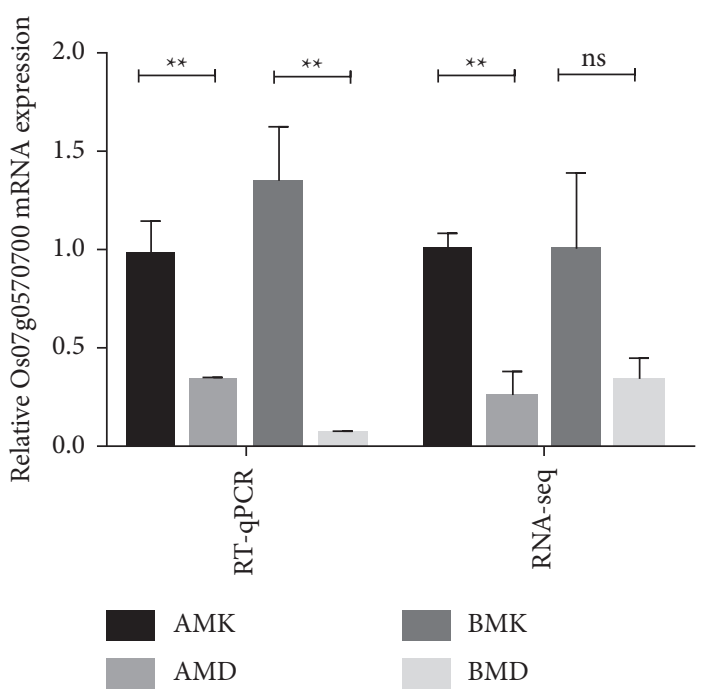

(h)

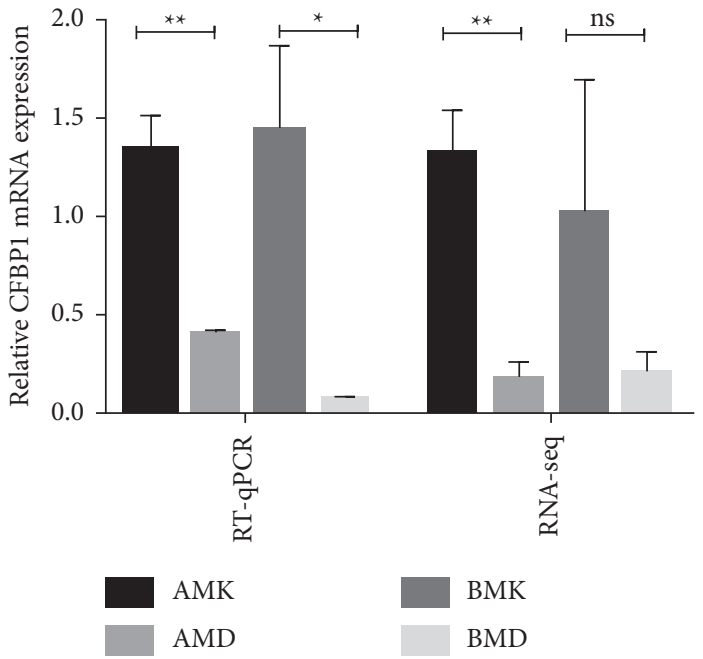

(j)

FIgURE 3: Comparative analysis of RT-qPCR and RNA-seq outcomes of expression patterns of 10 interested genes (i.e., PEX11-4 (a), WNK3 (b), CLPB1 (c), GDCSH (d), HSFB2C (e), LAC6 (f), Os06g0196300 (g), Os07g0570700 (h), ADI1 (i), and CFBP1 (j)) in roots of XWX and YZX.

[25, 26], HCF136 [27], ALDP [28], TPI [29, 30], and HSP17.4 [31] have been found to be implicated in Cd response. ADI1 has also been reported to be related to $\mathrm{Cd}$ stress in Agaricus brasiliensis [32]. Cd exposure could lead to the increase of CLPB expression level and delay of cell division in Escherichia coli $[33,34]$. Additionally, our analysis revealed that 6 genes (LAC6, CFBP1, CLPB1, WNK3, OsJ_24808, and ADI1) had a potential ion binding activity. Probable serine/threonine-protein kinase WNK3 belongs to the WNK family, which plays vital roles in plant growth and development and stress responses $[35,36]$. A recent study showed that WNK9 (a member of the WNK family) overexpression could improve arsenite (a heavy mental) tolerance in transgenic Arabidopsis [37]. Furthermore, annotation analysis revealed that 3 genes (LAC6, OsJ_019618, and PEX11-4) were related to oxidative stress.
Oxidative stress has been found to be closely linked with Cd tolerance and Cd-induced injury and toxicity [38-40]. For instance, molybdenum could relieve $\mathrm{Cd}$-induced toxicity and potentiate $\mathrm{Cd}$ tolerance by restricting $\mathrm{Cd}$ uptake, reducing oxidative stress, and improving antioxidant defense responses [41]. These data suggested that these genes might function as crucial players in the response to Cd stress.

Cao et al. showed that 706 transcripts were specially expressed in the roots of the mutant Indica rice (Icd1 group), which had an extremely low Cd accumulation in root and shoot under Cd exposure [42]. Moreover, 987 transcripts were specially expressed in the roots of wild-type rice (WT group) under Cd exposure [42]. These transcripts might be implicated in the difference in Cd accumulation in wild-type and mutanttype Icd1 rice. Combined with our data and Cao's outcomes, we found that 4 transcripts (BGIOSGA004865 (CFBP1), 


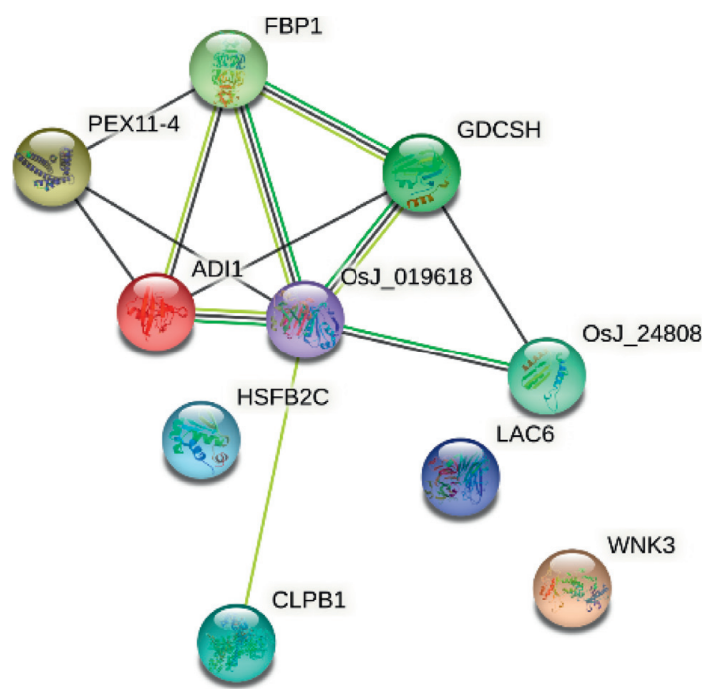

Figure 4: Interaction network of 10 interested genes. The yellow line represents that the potential interaction between proteins was obtained by text mining. The black line denotes that the potential interaction between proteins was identified by coexpression analysis. The green line means that the potential interaction between proteins was predicted based on gene neighborhood relationships. PPI analysis suggested that PEX11-4 could interact with CFBP1 (FBP1), ADI1, and Os06g0196300 (OsJ_019618). There was a potential interaction between CFBP1 (FBP1) and PEX11-4/ADI1/Os06g0196300 (OsJ_019618)/GDCSH. GDCSH coud interact with CFBP1 (FBP1)/ADI1/Os06g0196300 (OsJ_019618)/Os07g0570700 (OsJ_24808). Os07g0570700 (OsJ_24808) could interact with GDCSH/Os06g0196300 (OsJ_019618). Os06g0196300 (OsJ_019618) could interact with ADI1/PEX11-4/CFBP1 (FBP1)/GDCSH/Os07g0570700 (OsI_24808)/CLPB1. ADI1 could interact with PEX11-4/CFBP1 (FBP1)/GDCSH/Os06g0196300 (OsJ_019618). CLPB1 could interact with Os06g0196300 (OsJ_019618).

BGIOSGA017856 (Os05g0480200), BGIOSGA031509 (GSTU6), and BGIOSGA033278 (GDCSH)) that were specially expressed in the Icd1 group were differentially expressed in the AMD vs. AMK group. Also, 11 transcripts (BGIOSGA000239 (SPS1), BGIOSGA000945 (LEA14), BGIOSGA001233 (ABCG36), BGIOSGA005931 (YSL2), BGIOSGA006919 (SAT1), BGIOSGA007802 (CKX6), BGIOSGA011045 (HSP17.4), BGIOSGA014421 (AOX1B), BGIOSGA021670 (OPR1), BGIOSGA033315 (GSTU6), and BGIOSGA033325 (GSTU6)) or 6 transcripts (BGIOSGA000239 (SPS1), BGIOSGA006919 (SAT1), BGIOSGA009154 (HSP18.6), BGIOSGA011045 (HSP17.4), BGIOSGA021670 (OPR1), and BGIOSGA033325 (GSTU6)) that were specially expressed in the WT group were differentially expressed in the AMD vs. AMK or BMD vs. BMK group, respectively. Among these genes, 2 genes (GSTU6 and HSP17.4) were annotated to be involved in $\mathrm{Cd}$ response by the GO database. Moreover, GSTU6 has been found to be notably upregulated in roots of rice under Cd stress [43]. HSP17.4 expression was markedly increased in Arabidopsis thaliana roots after Cd exposure [31]. SPS1 was notably downregulated in response to Cd exposure in Chlorella sorokiniana [44]. ABCG36 (PDR9) expression was markedly increased in the roots of rice after a short exposure to Cd $[45,46]$. ABCG36 knockout promoted root Cd accumulation and potentiated $\mathrm{Cd}$ sensitivity in rice [45]. The YSL2 expression level was noticeably increased in the root and shoot of rice in response to Cd stress and YSL2 might be related to $\mathrm{Cd}$ uptake transport $[47,48]$. These transcripts might play vital roles in the response to $\mathrm{Cd}$ stress in rice.

\section{Conclusion}

Taken together, a host of $\mathrm{Cd}$ response-related transcripts were identified in the roots of YZX and XWX at the booting stage. Some transcripts that might be associated with the difference in the root Cd-responsive ability of these two rice cultivars were screened out. Moreover, our data suggested that ADI1, CFBP1, PEX11-4, OsJ_019618, OsJ_24808, GDCSH, CLPB1, and WNK3 might play vital roles in regulating $\mathrm{Cd}$ response and $\mathrm{Cd}$ tolerance. An in-depth insight into the genetic and molecular mechanisms underlying root $\mathrm{Cd}$ stress response difference in YZX and XWX at the booting stage might contribute to the development of new strategies that can improve rice yield and reduce $\mathrm{Cd}$ harm to rice and humans.

\section{Data Availability}

The data supporting these results are available from the corresponding author upon request.

\section{Conflicts of Interest}

The authors declare that they have no conflicts of interest.

\section{Acknowledgments}

This research was financially supported by the National Natural Science Foundation of China (no. 41907032), the Science and Technology Support Project of Guizhou 
Province (nos. 2018.2295 and 2019.2305), and the Key Scientific and Technological Special Project of Changsha City in China (no. kq200602019.230526).

\section{Supplementary Materials}

Supplementary Table 1: differentially expressed transcripts in the AMD vs. AMK group. Supplementary Table 2: differentially expressed transcripts in the BMD vs. BMK group. Supplementary Table 3: interaction of proteins encoded by differentially expressed genes in the AMD vs. AMK group. Supplementary Table 4: interaction of proteins encoded by differentially expressed genes in the BMD vs. BMK group. Supplementary Table 5: the node degree of each gene in Supplementary Table 3. Supplementary Table 6: the node degree of each gene in Supplementary Table 4. Supplementary Table 7: differentially expressed transcripts related to $\mathrm{Cd}$ responses in the AMD vs. AMK group, which was annotated by the GO database. Supplementary Table 8: GOannotated Cd-responsive transcripts, which were also differentially expressed in the BMD vs. BMK group. Supplementary Table 9: Venn analysis outcomes for differentially expressed transcripts related to Cd responses in the AMD vs. AMK and BMD vs. BMK groups. Supplementary Table 10: transcripts that were markedly upregulated or downregulated in the BMD vs. BMK group, but not in the AMD vs. AMK group. Supplementary Table 11: transcripts that were notably upregulated or downregulated in the AMD vs. AMK group, but not in the BMD vs. BMK group. Supplementary Table 12: the information of transcripts in Supplementary Tables 10 and 11. Supplementary Table 13: KEGG enrichment analysis for genes in Supplementary Table 12. Supplementary Table 14: the interaction, enrichment, and annotation analysis of 10 interested genes. Supplementary Figure 1: identification of differentially expressed transcripts in AMD vs. AMK and BMD vs. BMK groups. (A) The heat map of differentially expressed transcripts in the AMD vs. AMK group. (B) The heat map of differentially expressed transcripts in the BMD vs. BMK group. Supplementary Figure 2: construction of interaction networks of dysregulated genes. (A) Interaction networks of differentially expressed genes in the AMD vs. AMK group. (B) Interaction networks of differentially expressed genes in the BMD vs. BMK group. (Supplementary Materials)

\section{References}

[1] C. Marco, D. L. Maria, G. Cádiz, and S. C. Antonio, "Xtraction and analysis of phenolic compounds in rice: a review," Molecules, vol. 23, no. 11, p. 2890, 2018.

[2] L. Nie and S. Peng, "Rice production in China," Rice Production Worldwide, , pp. 33-52, Springer, 2017.

[3] N. Li, R. Xu, P. Duan, and Y. Li, "Control of grain size in rice," Plant Reproduction, vol. 31, no. 3, pp. 237-251, 2018.

[4] P. Wang, H. Chen, P. M. Kopittke, and F.-J. Zhao, "Cadmium contamination in agricultural soils of China and the impact on food safety," Environmental Pollution, vol. 249, pp. 1038-1048, 2019.

[5] Q. Yang, Z. Li, X. Lu, Q. Duan, L. Huang, and J. Bi, “A review of soil heavy metal pollution from industrial and agricultural regions in China: pollution and risk assessment," The Science of the Total Environment, vol. 642, pp. 690-700, 2018.

[6] M. Zou, S. Zhou, Y. Zhou, Z. Jia, T. Guo, and J. Wang, "Cadmium pollution of soil-rice ecosystems in rice cultivation dominated regions in China: a review," Environmental Pollution, vol. 280, Article ID 116965, 2021.

[7] J. Chen, W. Zou, L. Meng, X. Fan, G. Xu, and G. Ye, "Advances in the uptake and transport mechanisms and QTLs mapping of cadmium in rice," International Journal of Molecular Sciences, vol. 20, no. 14, p. 3417, 2019.

[8] W.-E. Song, S.-B. Chen, J.-F. Liu, L. Song, N. Li, and B. Liu, "Variation of $\mathrm{Cd}$ concentration in various rice cultivars and derivation of cadmium toxicity thresholds for paddy soil by species-sensitivity distribution," Journal of Integrative Agriculture, vol. 14, no. 9, pp. 1845-1854, 2015.

[9] G. Genchi, M. S. Sinicropi, G. Lauria, A. Carocci, and A. Catalano, "The effects of cadmium toxicity," International Journal of Environmental Research and Public Health, vol. 17, no. 11, p. 3782, 2020.

[10] B. Hussain, M. N. Ashraf, R. Shafeeq-ur-Rahman, A. Abba, and M. Farooq, "Cadmium stress in paddy fields: effects of soil conditions and remediation strategies," The Science of the Total Environment, vol. 754, Article ID 142188, 2021.

[11] J. J. V. Branca, G. Morucci, and A. Pacini, "Cadmium-induced neurotoxicity: still much ado," Neural Regeneration Research, vol. 13, no. 11, pp. 1879-1882, 2018.

[12] L. Sun, X. Xu, Y. Jiang et al., "Genetic diversity, rather than cultivar type, determines relative grain Cd accumulation in hybrid rice," Frontiers of Plant Science, vol. 7, p. 1407, 2016.

[13] G. Duan, G. Shao, Z. Tang et al., "Genotypic and environmental variations in grain cadmium and arsenic concentrations among a panel of high yielding rice cultivars," Rice, vol. 10, no. 1, p. 9, 2017.

[14] S. Naidoo, E. A. Visser, L. Zwart, Y. D. Toit, V. Bhadauria, and L. S. Shuey, "Dual RNA-sequencing to elucidate the plantpathogen duel," Current Issues in Molecular Biology, vol. 27, pp. 127-142, 2018.

[15] S. Jones, A. Baizan-Edge, S. MacFarlane, and L. Torrance, "Viral diagnostics in plants using next generation sequencing: computational analysis in practice," Frontiers of Plant Science, vol. 8, p. 1770, 2017.

[16] A. Liu, Z. Zhou, Y. Yi, and G. Chen, "Transcriptome analysis reveals the roles of stem nodes in cadmium transport to rice grain,” BMC Genomics, vol. 21, no. 1, p. 127, 2020.

[17] H. Chen, X. Chen, J. Tian et al., "Development of gene-based SSR markers in rice bean (vigna umbellata L.) based on transcriptome data," PLoS One, vol. 11, no. 3, Article ID e0151040, 2016.

[18] M. Israël Prince, M. François, S. Sun, and X. Lin Fan, "Effects of alkaline fertilizer and rice cultivation (Oryza sativa L.) on remediation of soils polluted with cadmium (Cd)," Journal of Applied Biosciences, vol. 157, pp. 16182-16193, 2021.

[19] L. Ge, L. Cang, J. Yang, and D. Zhou, "Effects of root morphology and leaf transpiration on Cd uptake and translocation in rice under different growth temperature," Environmental Science and Pollution Research, vol. 23, no. 23, pp. 24205-24214, 2016.

[20] L.-Q. Ge, L. Cang, H. Liu, and D.-M. Zhou, "Effects of warming on uptake and translocation of cadmium (Cd) and copper $(\mathrm{Cu})$ in a contaminated soil-rice system under Free Air Temperature Increase (FATI)," Chemosphere, vol. 155, pp. 1-8, 2016.

[21] H. Zhou, W. Zhu, W.-T. Yang et al., "Cadmium uptake, accumulation, and remobilization in iron plaque and rice 
tissues at different growth stages," Ecotoxicology and Environmental Safety, vol. 152, pp. 91-97, 2018.

[22] W. Ali, K. Mao, H. Zhang et al., "Comprehensive review of the basic chemical behaviours, sources, processes, and endpoints of trace element contamination in paddy soil-rice systems in rice-growing countries," Journal of Hazardous Materials, vol. 397, Article ID 122720, 2020.

[23] R. Khanam, A. Kumar, A. K. Nayak et al., "Metal(loid)s (As, $\mathrm{Hg}$, Se, $\mathrm{Pb}$ and $\mathrm{Cd}$ ) in paddy soil: bioavailability and potential risk to human health," The Science of the Total Environment, vol. 699, Article ID 134330, 2020.

[24] S. Sharma, I. Kaur, and A. K. Nagpal, "Contamination of rice crop with potentially toxic elements and associated human health risks-a review," Environmental Science and Pollution Research, vol. 28, no. 10, pp. 12282-12299, 2021.

[25] M. Y. Lee and H. W. Shin, "Cadmium-induced changes in antioxidant enzymes from the marine alga Nannochloropsis oculata," Journal of Applied Phycology, vol. 15, no. 1, pp. 13-19, 2003.

[26] M. Jozefczak, S. Bohler, H. Schat et al., "Both the concentration and redox state of glutathione and ascorbate influence the sensitivity of arabidopsis to cadmium," Annals of Botany, vol. 116, no. 4, pp. 601-612, 2015.

[27] W.-L. Huang, F.-L. Wu, H.-Y. Huang et al., "Excess copperinduced alterations of protein profiles and related physiological parameters in citrus leaves," Plants, vol. 9, no. 3, p. 291, 2020.

[28] R. Yu, Y. Ma, Y. Li, X. Li, C. Du, and G. Shi, "Comparative transcriptome analysis revealed key factors for differential cadmium transport and retention in roots of two contrasting peanut cultivars," BMC Genomics, vol. 19, no. 1, p. 938, 2018.

[29] Z.-W. Cheng, Z.-Y. Chen, X. Yan, Y.-W. Bian, X. Deng, and Y.-M. Yan, "Integrated physiological and proteomic analysis reveals underlying response and defense mechanisms of Brachypodium distachyon seedling leaves under osmotic stress, cadmium and their combined stresses," Journal of Proteomics, vol. 170, pp. 1-13, 2018.

[30] C.-L. Ge, Z.-G. Wang, D.-Z. Wan et al., "Proteomic study for responses to cadmium stress in rice seedlings," Rice Science, vol. 16, no. 1, pp. 33-44, 2009.

[31] M. Weber, A. Trampczynska, and S. Clemens, "Comparative transcriptome analysis of toxic metal responses in Arabidopsis thaliana and the Cd2+-hypertolerant facultative metallophyte Arabidopsis halleri," Plant, Cell and Environment, vol. 29, no. 5, pp. 950-963, 2006.

[32] P.-H. Liu, Z.-X. Huang, X.-H. Luo et al., "Comparative transcriptome analysis reveals candidate genes related to cadmium accumulation and tolerance in two almond mushroom (Agaricus brasiliensis) strains with contrasting cadmium tolerance," PLoS One, vol. 15, no. 9, Article ID e0239617, 2020.

[33] Z. Khan, M. A. Nisar, S. Z. Hussain, M. N. Arshad, and A. Rehman, "Cadmium resistance mechanism in Escherichia coli $\mathrm{P} 4$ and its potential use to bioremediate environmental cadmium," Applied Microbiology and Biotechnology, vol. 99, no. 44, pp. 10745-10757, 2015.

[34] A. Wang and D. E. Crowley, "Global gene expression responses to cadmium toxicity in Escherichia coli," Journal of Bacteriology, vol. 187, no. 9, pp. 3259-3266, 2005.

[35] R. Manuka, A. A. Saddhe, and K. Kumar, "Genome-wide identification and expression analysis of WNK kinase gene family in rice," Computational Biology and Chemistry, vol. 59, no. Pt A, pp. 56-66, 2015.
[36] A. A. Saddhe, S. B. Karle, T. Aftab, and K. Kumar, "With no lysine kinases: the key regulatory networks and phytohormone cross talk in plant growth, development and stress response," Plant Cell Reports, vol. 40, no. 11, pp. 2097-2109, 2021.

[37] R. Manuka, A. A. Saddhe, A. K. Srivastava, K. Kumar, and S. Penna, "Overexpression of rice OsWNK9 promotes arsenite tolerance in transgenic Arabidopsis plants," Journal of Biotechnology, vol. 332, pp. 114-125, 2021.

[38] S. Nemmiche, "Oxidative signaling response to cadmium exposure," Toxicological Sciences: An Official Journal of the Society of Toxicology, vol. 156, no. 1, pp. 4-10, 2017.

[39] C. Loix, M. Huybrechts, J. Vangronsveld, M. Gielen, E. Keunen, and A. Cuypers, "Reciprocal interactions between cadmium-induced cell wall responses and oxidative stress in plants," Frontiers of Plant Science, vol. 8, p. 1867, 2017.

[40] A. Cuypers, M. Plusquin, T. Remans et al., "Cadmium stress: an oxidative challenge," Biometals, vol. 23, no. 5, pp. 927-940, 2010.

[41] M. Imran, S. Hussain, M. A. El-Esawi et al., "Molybdenum supply alleviates the cadmium toxicity in fragrant rice by modulating oxidative stress and antioxidant gene expression," Biomolecules, vol. 10, no. 11, p. 1582, 2020.

[42] Z. Z. Cao, X. Y. Lin, Y. J. Yang, M. Y. Guan, P. Xu, and M. X. Chen, "Gene identification and transcriptome analysis of low cadmium accumulation rice mutant (lcd1) in response to cadmium stress using MutMap and RNA-seq," BMC Plant Biology, vol. 19, no. 1, p. 250, 2019.

[43] Y. Huang, H. Chen, J. R. Reinfelder et al., "A transcriptomic (RNA-seq) analysis of genes responsive to both cadmium and arsenic stress in rice root," The Science of the Total Environment, vol. 666, pp. 445-460, 2019.

[44] N. Ding, L. Wang, Y. Kang et al., "The comparison of transcriptomic response of green microalga Chlorella sorokiniana exposure to environmentally relevant concentration of cadmium(II) and 4-n-nonylphenol," Environmental Geochemistry and Health, vol. 42, no. 9, pp. 2881-2894, 2020.

[45] S. Fu, Y. Lu, X. Zhang et al., "The ABC transporter ABCG36 is required for cadmium tolerance in rice," Journal of Experimental Botany, vol. 70, no. 20, pp. 5909-5918, 2019.

[46] A. Moons, "Ospdr9, which encodes a PDR-type ABC transporter, is induced by heavy metals, hypoxic stress and redox perturbations in rice roots1," FEBS Letters, vol. 553, no. 3, pp. 370-376, 2003.

[47] T. Guha, S. Barman, A. Mukherjee, and R. Kundu, "Nanoscale zero valent iron modulates $\mathrm{Fe} / \mathrm{Cd}$ transporters and immobilizes soil Cd for production of Cd free rice," Chemosphere, vol. 260, Article ID 127533, 2020.

[48] X. Li, D. Chen, B. Li, Y. Yang, and Y. Yang, "Combined transcriptomic, proteomic and biochemical approaches to identify the cadmium hyper-tolerance mechanism of turnip seedling leaves," Environmental Science and Pollution Research, vol. 28, no. 18, pp. 22458-22473, 2021. 